\title{
In-plane free vibration analysis of an annular disk with point elastic support
}

\author{
S. Bashmal*, R. Bhat and S. Rakheja \\ Department of Mechanical and Industrial Engineering, Concordia University, 1455 De Maisonneuve Blvd. W., \\ Montreal, QC, H3G 1M8, Canada
}

Received 11 April 2009

Revised 6 February 2010

\begin{abstract}
In-plane free vibrations of an elastic and isotropic annular disk with elastic constraints at the inner and outer boundaries, which are applied either along the entire periphery of the disk or at a point are investigated. The boundary characteristic orthogonal polynomials are employed in the Rayleigh-Ritz method to obtain the frequency parameters and the associated mode shapes. Boundary characteristic orthogonal polynomials are generated for the free boundary conditions of the disk while artificial springs are used to account for different boundary conditions. The frequency parameters for different boundary conditions of the outer edge are evaluated and compared with those available in the published studies and computed from a finite element model. The computed mode shapes are presented for a disk clamped at the inner edge and point supported at the outer edge to illustrate the free in-plane vibration behavior of the disk. Results show that addition of point clamped support causes some of the higher modes to split into two different frequencies with different mode shapes.
\end{abstract}

Keywords: Rayleigh-Ritz method, in-plane free vibration, natural frequencies, boundary characteristic orthogonal polynomials

\section{Introduction}

The vibration characteristics of circular disks subject to classical boundary conditions have been widely reported. These have provided vast amount of information on out-of-plane vibration behavior of disks. In-plane vibration of circular disks, however, have been gaining increasing attention only in recent years, in view of its potential applications in various practical problems including the vibration of railway wheels, disk brakes and hard disk drives [1-3]. Furthermore, the in-plane dynamics of rotating disks is considered to be of practical importance in view of its application in rolling noise emission arising from to wheel-rail interactions [4].

The first attempt to tackle the problem of vibration within the plane of disks was by Love [5], who derived the essential equations of motion and presented the general solution for a thin circular disk with free edge conditions. Onoe [6] evaluated the in-plane vibration of disks using numerical analysis and presented the frequency equations corresponding to the different modes. Holland [7] presented the frequency parameters and eigenmodes for a wide range of Poisson's ratios and investigated the response due to an in-plane force. Another study evaluated the frequency parameters and associated mode shapes of in-plane vibration of solid disks clamped at the outer edge using assumed deflection modes in terms of trigonometric and Bessel functions [8]. More recently, Park [9] obtained an exact frequency equation for a solid disk clamped at the outer edge.

The above studies were limited to in-plane free vibration of solid disks. Such analyses of annular disks have been addressed only in a few studies. Irie et al. [10] evaluated the natural frequencies of in-plane vibration of annular disks using transfer matrix formulation with combinations of free and clamped conditions at the inner and outer edges,

\footnotetext{
*Corresponding author. E-mail: s_bashm@encs.concordia.ca.
} 


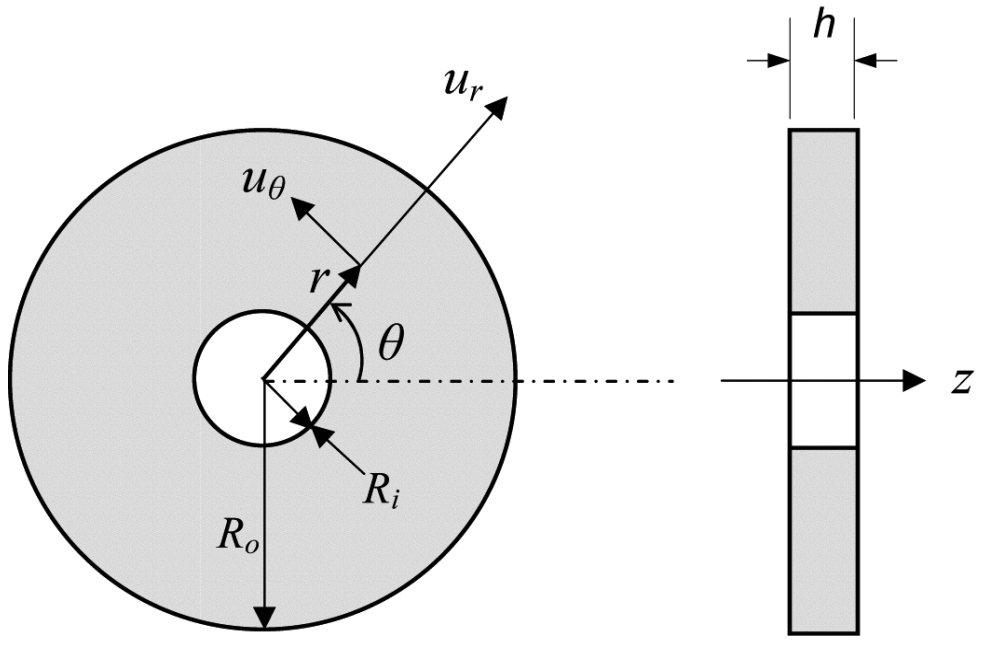

Fig. 1. Geometry and coordinate system used for in-plane vibration analysis of an annular disk.

while the corresponding mode shapes were not presented. Ambati et al. [11] presented a generalized formulation for in-plane vibration analysis of annular disks and extended the application to solid disks as well as thin rings by varying size of the opening. The authors also illustrated verification of analytical results through experiments.

The reported studies have considered either clamped or free boundary conditions. A perfectly clamped condition, however, may not exist in practice. Flexible boundary conditions may be considered more representative of practical situations. The approach of employing elastic springs distributed uniformly around the edge has been extensively used in problems related to out-of-plane vibrations of circular plates [12-14] and cylindrical shells [15]. The advantage of using this method can be evident when couplings between two or more elastic components are involved. A rigid joint between two components can be approximated by inserting artificial springs of large stiffness, while flexible joints may be simulated by assigning the representative stiffness value for the joint [15]. Several studies have also considered the effect of partial constraints and stiffness non-uniformity [16-19] on the out-of-plane vibrations of plates. It is found that partial constraints cause frequency parameters to split into symmetric and anti-symmetric frequencies corresponding to each mode [16]. These frequency parameters, however, were identical when uniform boundary conditions were considered.

The studies on in-plane vibration of disks, however, have not yet considered the effects of partial constraints, while the effect of flexible boundary conditions have been reported by the authors [22]. Consideration of circular disks with either a point or a patch constraint would be more practical for study of in-plane vibration in many situations such as automobile wheel/tire assembly and railway wheels. The dynamic railway wheel and track interaction in the presence of wheel flat defects would be of particular interest in the context of in-plane wheel vibration, which may contribute to dynamic wheel impact loads and noise emission [4]. The present study is an attempt to understand the effect of partial constraints and flexible boundary conditions on the in-plane free vibration of circular disks. The boundary characteristic orthogonal polynomials [20] are employed as assumed deflection functions in the RayleighRitz method to obtain the natural frequencies and associated mode shapes. The partial constraint condition was also achieved by introducing artificial springs along a portion of the free edge. The frequency parameters are tabulated and the mode shapes are depicted to illustrate the in-plane free vibration behavior of circular disks. The results are compared with the reported data and those obtained from finite element analysis to illustrate the validity of the present method.

\section{Theory}

Consider an annular disk with outer radius $R_{o}$, inner radius $R_{i}$ and thickness $h$, as shown in Fig. 1. It is assumed that the disk is isotropic with mass density $\rho$, Young's modulus $E$ and Poisson's ratio $v$. The radial and circumferential 
displacement components of a point in the plane of the annular disk are denoted by $u_{r}$ and $u_{\theta}$, respectively. The expression for the strain energy of the disk in polar coordinates $(r, \theta)$ is derived from the constitutive laws and strain-displacement relations. Introducing the non-dimensional parameter, $\xi=r / R_{o}$, the maximum strain energy can be expressed in terms of the displacements as:

$$
\begin{aligned}
W= & \frac{1}{2} \int_{0}^{2 \pi} \int_{\beta}^{1} \frac{E h}{1-v^{2}}\left\{\left(\frac{\partial u_{r}}{\partial \xi}\right)^{2}+2 v\left(\frac{u_{r}}{\xi} \frac{\partial u_{r}}{\partial \xi}+\frac{1}{\xi} \frac{\partial u_{\theta}}{\partial \theta} \frac{\partial u_{r}}{\partial \xi}\right)+\left(\frac{u_{r}}{\xi}\right)^{2}\right. \\
& \left.+2 \frac{u_{r}}{\xi^{2}} \frac{\partial u_{\theta}}{\partial \theta}+\frac{1}{\xi^{2}}\left(\frac{\partial u_{\theta}}{\partial \theta}\right)^{2}+\frac{1}{2}(1-v)\left(\frac{1}{\xi} \frac{\partial u_{r}}{\partial \theta}+\frac{\partial u_{\theta}}{\partial \xi}-\frac{u_{\theta}}{\xi}\right)^{2}\right\} \xi d \xi d \theta
\end{aligned}
$$

Similarly, the kinetic energy of the disk can be expressed as:

$$
T=\frac{1}{2} \int_{0}^{2 \pi} \int_{\beta}^{1}\left(\dot{u}_{r}^{2}+\dot{u}_{\theta}^{2}\right) h \rho R_{0}^{2} \xi d \xi d \theta,
$$

where $W$ and $T$ are the strain and kinetic energy functions, respectively and $\beta=R_{i} / R_{o}$ is the radius ratio between inner and outer radii of the disk. The free in-plane vibration of the disk is assumed to have sinusoidal variations along the circumferential axis of the disk, and may be expressed in the form:

$$
\begin{aligned}
& u_{r}(\xi, \theta, t)=\sum_{n=0}^{\infty} U_{c, n}(\xi, t) \cos (n \theta)+U_{s, n}(\xi, t) \sin (n \theta), \\
& u_{\theta}(\xi, \theta, t)=\sum_{n=0}^{\infty} V_{c, n}(\xi, t) \cos (n \theta)+V_{s, n}(\xi, t) \sin (n \theta),
\end{aligned}
$$

where $n=0,1,2, \ldots$, is the circumferential wave number or nodal diameter number, and $U$ and $V$ are radial and circumferential deflection functions, respectively, expressed as linear combinations of assumed deflection modes in the form of boundary characteristic orthogonal polynomials [20]:

$$
\begin{array}{ll}
U_{c, n}(\xi, t) & =\sum_{m=0}^{\infty} \phi_{m}(\xi) \bar{U}_{c, m n}(t), \quad U_{s, n}(\xi, t)=\sum_{m=0}^{\infty} \phi_{m}(\xi) \bar{U}_{s, m n}(t), \\
V_{c, n}(\xi, t) & =\sum_{m=0}^{\infty} \phi_{m}(\xi) \bar{V}_{c, m n}(t), \quad V_{s, n}(\xi, t)=\sum_{m=0}^{\infty} \phi_{m}(\xi) \bar{V}_{s, m n}(t)
\end{array}
$$

where the first subscripts of functions $U$ and $V, c$ and $s$ refer to cosine and sine components of the deflections respectively, $\bar{U}$ and $\bar{V}$ are the deflection coefficients, and $\phi_{m}(\xi)$ is the assumed deflection shapes corresponding to mode $m$.

The first deflection mode, $\phi_{m}(\xi)$ is generally chosen as a polynomial of the lowest degree that satisfies the geometric boundary conditions. In the present study, this starting function is selected as, $\phi_{0}(\xi)=1$. The successive polynomials are generated using the recurrence relations [20], such that:

$$
\begin{aligned}
& \phi_{1}(\xi)=\left(\xi-b_{0}\right) \phi_{0}(\xi) \\
& \phi_{k+1}(\xi)=\left(\xi-b_{k}\right) \phi_{k}(\xi)-c_{k} \phi_{k-1}(\xi), \quad k \geqslant 1
\end{aligned}
$$

where 


$$
\begin{aligned}
b_{k} & =\int_{\beta}^{1} \xi^{2} \phi_{k}^{2}(\xi) d \xi / \int_{\beta}^{1} \xi \phi_{k}^{2}(\xi) d \xi, \quad k \geqslant 0 \\
c_{k} & =\int_{\beta}^{1} \xi^{2} \phi_{k}(\xi) \phi_{k-1}(\xi) d \xi / \int_{\beta}^{1} \xi \phi_{k-1}^{2}(\xi) d \xi, \quad k \geqslant 1 .
\end{aligned}
$$

Substituting Eqs (3) and (4) into (1) and (2), and equating the maximum kinetic and potential energies yields the Rayleigh's quotient $\Omega^{2}$, where $\Omega^{2}=\rho \omega^{2} R_{0}^{2}\left(1-v^{2}\right) / E$. Application of the condition of stationarity with respect to the arbitrary coefficients, $\bar{U}_{c, m n}, \bar{U}_{s, m n}, \bar{V}_{c, m n}$ and $\bar{V}_{s, m n}$, of the form:

$$
\begin{aligned}
\frac{\partial \Omega^{2}}{\partial \bar{U}_{c, m n}}=0 ; & \frac{\partial \Omega^{2}}{\partial \bar{U}_{s, m n}}=0 ; \\
\frac{\partial \Omega^{2}}{\partial \bar{V}_{c, m n}}=0 ; & \frac{\partial \Omega^{2}}{\partial \bar{V}_{s, m n}}=0 ;
\end{aligned}
$$

results in the eigenvalue problem, as:

$$
\left([K]-\Omega^{2}[M]\right)\left\{\begin{array}{c}
\bar{U}_{c, m n} \\
\bar{U}_{s, m n} \\
\bar{V}_{c, m n} \\
\bar{V}_{s, m n}
\end{array}\right\}=\{0\}
$$

The eigenvectors in the above formulation include both the cosine and sine components of the radial and circumferential displacements, where $[K]$ and $[M]$ are $(4 \times m \times n)$ nondimensional stiffness and mass square matrices, respectively. Due to the nature of couplings between the modes of non-rotating disks, the system of equations can be decomposed into two subsets, each of size $(2 \times m \times n)$ [21]. The solution of Eq. (9) reveals that the radial cosine components $\bar{U}_{c, m n}$ are coupled with the corresponding circumferential sine components $\bar{V}_{s, m n}$ only, while the radial sine component $\bar{U}_{s, m n}$ are coupled with the circumferential cosine components $\bar{V}_{c, m n}$ only. The couplings between the various modes and their effects are described in the subsequent sections. The formulations of the stiffness and mass matrices are thus presented considering only one subset of equations. Furthermore, the subscripts $c$ and $s$ corresponding to Conference: Models of Dementia: The Good, the Bad and the Future cosine and sine components are omitted for the purpose of brevity. The stiffness matrix $[K]$ is formulated as:

$$
[K]=\left[\begin{array}{ll}
{[U U]} & {[U V]} \\
{[V U]} & {[V V]}
\end{array}\right],
$$

where

$$
\begin{aligned}
& (U U)_{i j}=\int_{\beta}^{1}\left(\begin{array}{l}
\xi \phi_{i}^{\prime} \phi_{j}^{\prime}+v\left(\phi_{i}^{\prime} \phi_{j}+\phi_{i} \phi_{j}^{\prime}\right) \\
+\frac{\phi_{i} \phi_{j}}{\xi}+\frac{1-v}{2} \frac{n^{2}}{\xi} \phi_{i} \phi_{j}
\end{array}\right) d \xi ;(V V)_{i j}=\int_{\beta}^{1}\left(\begin{array}{c}
\frac{n^{2}}{\xi} \phi_{i} \phi_{j}+\frac{1-v}{2} \xi \phi_{i}^{\prime} \phi_{j}^{\prime} \\
+\frac{v-1}{2}\left(\phi_{i}^{\prime} \phi_{j}+\phi_{i} \phi_{j}^{\prime}\right)+\frac{1-v}{2} \frac{\phi_{i} \phi_{j}}{\xi}
\end{array}\right) d \xi \\
& (U V)_{i j}=\int_{\beta}^{1}\left(\begin{array}{l}
\frac{n}{\xi} \phi_{i} \phi_{j}+v n \phi_{i}^{\prime} \phi_{j} \\
+\frac{v-1}{2} n \phi_{i} \phi_{j}^{\prime}+\frac{1-v}{2} n \frac{\phi_{i} \phi_{j}}{\xi}
\end{array}\right) d \xi ; \\
& (V U)_{i j}=\int_{\beta}^{1}\left(\begin{array}{l}
\frac{n}{\xi} \phi_{i} \phi_{j}+v n \phi_{i} \phi_{j}^{\prime} \\
+\frac{v-1}{2} n \phi_{i}^{\prime} \phi_{j}+\frac{1-v}{2} n \frac{\phi_{i} \phi_{j}}{\xi}
\end{array}\right) d \xi ;
\end{aligned}
$$

and $\phi_{i}^{\prime}=\frac{d \phi}{d \xi}$.

The mass matrix $[M]$ is given as:

$$
[M]=\left[\begin{array}{ll}
{\left[m_{i i}\right]} & {[0]} \\
{[0]} & {\left[m_{i i}\right]}
\end{array}\right]
$$

where 


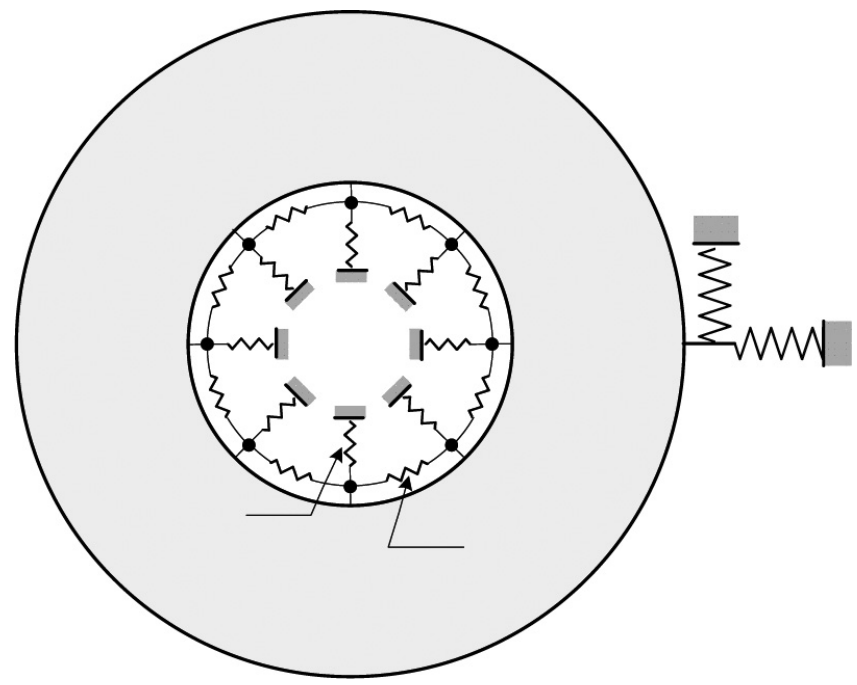

Fig. 2. Annular disk clamped at the inner edge and with elastic point support at the outer edge.

$$
m_{i i}=\int_{\beta}^{1} \phi_{i}^{2} \xi d \xi
$$

The mass matrix is diagonal because of the orthogonal property of the assumed deflection shapes. The frequency parameters can be obtained by solving the eigenvalues problem, Eq. (10).

\subsection{Boundary conditions}

A set of artificial springs are introduced at the free outer and inner edges to simulate the clamped and flexible boundary conditions. Very large values of the spring stiffness may be chosen to represent the clamped boundary condition. The artificial springs are distributed in the radial and circumferential directions on both the inner and outer edges of the disk, as shown in Fig. 2. The artificial springs can also be applied along a portion of the edge along the circumferential direction to realize a partial constraint or point support. The maximum strain energy stored in the distributed artificial springs at the inner and outer edges, $W_{d}$ can be derived from:

$$
\begin{aligned}
W_{d}= & \frac{1}{2} h\left[\int_{0}^{2 \pi} K_{r o}(\theta)\left[u_{r}\left(R_{o}, \theta, t\right)\right]^{2} R_{o} d \theta+\int_{0}^{2 \pi} K_{r i}(\theta)\left[u_{r}\left(R_{i}, \theta, t\right)\right]^{2} R_{i} d \theta\right. \\
& \left.\int_{0}^{2 \pi} K_{\theta o}(\theta)\left[u_{\theta}\left(R_{o}, \theta, t\right)\right]^{2} R_{o} d \theta+\int_{0}^{2 \pi} K_{\theta i}(\theta)\left[u_{\theta}\left(R_{i}, \theta, t\right)\right]^{2} R_{i} d \theta\right],
\end{aligned}
$$

where $K(\theta)$ represents radial and circumferential stiffness per unit length of the disk, subscripts ro and ri denote radial springs at the outer and inner edges, respectively, and $\theta \mathrm{o}$ and $\theta \mathrm{i}$ represent the respective springs along the circumferential directions. It is assumed that the artificial springs are symmetric about $\theta=0$. The spring stiffness $K(\theta)$ may thus be expressed by a Fourier cosine series, as:

$$
K(\theta)=\frac{a_{0}}{2}+\sum_{l=1}^{\infty} a_{l} \cos (l \theta),
$$

where

$$
a_{l}=\frac{1}{\pi} \int_{0}^{2 \pi} K(\theta) \cos (l \theta) d \theta \quad(l=0,1,2, \ldots \ldots . .) .
$$


A clamped boundary condition may be realized by assuming $K(\theta)$ as a constant in Eq. (11) and by selecting nearly infinite stiffness values.

For a disk supported at a point on the outer edge, the maximum potential energy $W_{p}$ can be derived as:

$$
W_{p}=\frac{1}{2} K_{p r} h\left[u_{r}\left(R_{0}, \theta_{0}, t\right)\right]^{2}+\frac{1}{2} K_{p \theta} h\left[u_{r}\left(R_{0}, \theta_{0}, t\right)\right]^{2},
$$

where $K_{p r}$ and $K_{p \theta}$ are the stiffness per unit length due to the point elastic support in the radial and circumferential directions, respectively, and $\theta_{0}$ is the circumferential coordinate of the point support.

The total strain energy is obtained by adding either Eq. (11) or 2.2 to Eq. (1), depending upon the constraint. The solution of the eigenvalue problem then yields the frequency parameters for the in-plane vibration of the annular disk subject to selected boundary and constraint conditions at the two free edges. For example, a clamped condition at the inner boundary and a free outer boundary can be realized by letting $K_{r i}, K_{\theta i} \approx \infty$ and $K_{r o}, K_{\theta o} \approx 0$. The validity of the clamped and flexible boundary conditions realized through distributed artificial springs has been illustrated in a recent study by the authors in the context of in-plane vibration of an annular and solid disk [22]. The frequency parameters were obtained under classical boundary conditions on the basis of analysis of only one subset of equations and compared with those reported in published studies.

\subsection{Nature of couplings}

The in-plane vibration modes exhibit complex couplings among them. Moreover, the couplings strongly depend upon the boundary conditions. The solution of the eigenvalue problem for different boundary conditions can provide significant insight into the nature of couplings between different modes and the effects of boundary conditions. It should be noted that the mass matrix is diagonal due to the orthogonal property of the assumed deflection shapes. The stiffness matrix is non-diagonal due to presence of derivatives of assumed deflection shapes and non-uniform constraint conditions. The couplings among different modes can thus be solely attributed to the stiffness matrix. As discussed earlier, the system of equations can be represented by those of two independent subsystems, associated with coupled radial cosine and circumferential sine, and radial sine and circumferential cosine modes, respectively. In the following discussion, these are referred to as even and odd subsystems with respect to the radial displacement. It must be noted that the even radial displacement is coupled with the odd circumferential displacement only. The odd and even subsystems, however, would be coupled in the case of rotating disks, which is not considered in this paper.

For different combinations of classical boundary conditions, the following observations related to mode couplings can be made through examination of the stiffness matrix:

- The modes with different nodal diameter number $(n)$ are uncoupled. The coupling exists between modes with different nodal circles $(m)$ within the same nodal diameter number $(n)$. Consequently, each set of modes corresponding to the same $n$ can be studied independently.

- For axisymmetric modes, $n=0$, the modes are either purely radial or purely circumferential. The even subsystem would yield axisymmetric radial modes, while the odd subsystem gives the axisymmetric circumferential modes. Since the variation of displacements with is zero they are treated as axisymmetric.

- For $n \geqslant 1$, the radial and circumferential displacements are coupled within the same nodal diameter number $n$. The frequency parameters of the odd subsystem are identical to those obtained for the even subsystem. The modes of the odd subsystem, however, exhibit a phase shift of $\pi /(2 n)$ with respect to their counterpart. It would thus be sufficient to compute the frequency parameters of one of the subsystems.

For a disk with non-uniform boundary conditions, nature of coupling between the modes would differ from those observed under classical boundary conditions, specifically:

- The modes associated with different nodal diameter number $n$ are coupled. The modes thus cannot be studied independently. Although the two subsystems could still be treated independently assuming symmetry about $\theta=0$, the resulting modes will be coupled within each subsystem.

- For axisymmetric modes, $n=0$, the odd subsystem yields free vibration responses that are different from those of the even subsystem. However, the modes are no longer purely radial or purely circumferential due to the effect of partial constraints. 
Table 1

Comparisons of frequency parameters $\Omega$ of in-plane vibration of an annular disk clamped at the inner edge $(v=0.3$, $\beta=0.2)$

\begin{tabular}{lccc}
\hline$(m, n)$ & Reference [10] & Present study & FE Model \\
\hline$(0,0)^{\theta}$ & 0.352 & 0.3526 & 0.3524 \\
$(0,1)$ & 0.919 & 0.9193 & 0.9193 \\
$(0,2)$ & 1.542 & 1.5416 & 1.5414 \\
$(1,1)$ & 2.121 & 2.1208 & 2.1208 \\
$(0,3)$ & 2.157 & 2.1570 & 2.1568 \\
$(0,0)^{r}$ & 2.204 & 2.2040 & 2.2039 \\
\hline
\end{tabular}

$r$ : pure radial mode; $\theta$ : pure circumferential mode.

Table 2

Comparisons of frequency parameters $\Omega$ of out-of-plane vibration of a solid disk $(v=0.3)$

\begin{tabular}{cccccc}
\hline & \multicolumn{2}{c}{$\begin{array}{c}\text { Solid disk with } \\
\text { point clamped }\end{array}$} & & \multicolumn{2}{c}{$\begin{array}{c}\text { Solid disk with point } \\
\text { simply-supported }\end{array}$} \\
\cline { 2 - 3 } \cline { 5 - 6 }$(\mathrm{m}, \mathrm{n})$ & Reference [16] & FE Model & & Reference [16] & FE Model \\
\hline$(0,0)$ & 0.737 & 0.714 & & 0 & 0 \\
$(0,1)$ & 1.943 & 1.932 & & 1.816 & 1.815 \\
$(0,2)$ & 2.904 & 2.896 & & 2.795 & 2.793 \\
$(1,0)$ & 3.227 & 3.236 & & 3.200 & 3.200 \\
$(0,3)$ & 3.630 & 3.627 & & 4.052 & 4.052 \\
\hline
\end{tabular}

- Although the even and odd subsystems remain uncoupled, they yield different values of the frequency parameters. The effect of partial elastic support can be considered similar to the gyroscopic effect due to rotation in which natural frequencies split into two different values. Similar effect has been reported by Narita and Leissa [16] and Eastep and Hemmig [23] for the case of out-of-plane vibrations.

\section{Results}

The frequency parameters and the corresponding mode shapes of the annular disk with different edge constraints are obtained by solving the eigenvalue problem in Eq. (2.1). The validity of the analysis approach has been demonstrated in a recent study by the authors on the in-plane vibrations of circular disks with free and clamped boundary conditions at the inner and outer edges [22]. Considering that the natural frequencies of in-plane vibration of circular disks with only point or partial supports are not available in the literature, they were obtained by finite-element analysis for comparison and validation of the analytical results. Table 1 presents the frequency parameters of in-plane vibration of an annular disk clamped at the inner edge and with free outer boundary, referred to as 'Clamped-Free'. The computed results corresponding to selected mode shapes are compared with those reported in [10] and verified with the finite element results. The comparison reveals very good agreement between the analytical, finite element model and the reported results, suggesting the validity of the finite element and the analytical models under classical boundary conditions.

Owing to the lack of published results on the in-plane free vibration of disks under partial or point supports, the validity of the finite element model under such constraints could only be established on the basis of the out-of-plane free vibration responses. For this purpose, the frequency parameters reported by Narita and Leissa [16] for the out-ofplane vibration of point-simply supported and point-clamped solid disks were considered, and compared with those obtained from the finite element model (Table 2). The comparisons suggest reasonably good agreements between the reported and finite element model results under two different point support conditions. From the reasonably good agreements illustrated in Tables 1 and 2, it is deduced that the finite element model can be considered to yield reasonably accurate in-plane free vibration characteristics under constraints involving point, partial or full supports.

The frequency parameters and mode shapes for the in-plane free vibration of the annular disk were obtained under four different support conditions: (i) Clamped inner edge with point clamp support at the outer edge, referred 
Table 3

Even frequency parameters $\Omega$ of In-plane vibration for annular disk ( $v=0.3, \beta=0.2)$

\begin{tabular}{|c|c|c|c|c|c|c|c|c|}
\hline \multirow{2}{*}{$\begin{array}{l}\begin{array}{l}\text { Constraint } \\
(\mathrm{m}, \mathrm{n})\end{array} \\
\end{array}$} & \multicolumn{2}{|c|}{ Clamped-point clamped } & \multicolumn{2}{|c|}{ Point clamped-clamped } & \multicolumn{2}{|c|}{ Free-point clamped } & \multicolumn{2}{|c|}{ Point clamped-free } \\
\hline & Present study & FE Model & Present study & FE Model & Present study & FE Model & Present study & FE Model \\
\hline$(0,1)$ & 0.9561 & 0.9548 & 2.1618 & 2.1576 & 0.3073 & 0.3031 & 0.3040 & 0.2808 \\
\hline$(0,2)$ & 1.605 & 1.5999 & 2.6399 & 2.6334 & 1.1868 & 1.1883 & 1.1633 & 1.1584 \\
\hline$(1,1)$ & 2.1349 & 2.135 & 3.3025 & 3.3055 & 1.6589 & 1.6645 & 1.6851 & 1.6850 \\
\hline$(0,3)$ & 2.1882 & 2.1882 & 3.7375 & 3.7351 & 1.8327 & 1.8304 & 1.8355 & 1.8284 \\
\hline$(0,0) r$ & 2.3097 & 2.2999 & 3.3944 & 3.3847 & 2.145 & 2.1471 & 2.0793 & 2.0845 \\
\hline
\end{tabular}

$r$ : pure radial mode; $\theta$ : pure circumferential mode.

Table 4

Odd frequency parameters $\Omega$ of In-plane vibration for annular disk $(v=0.3, \beta=0.2)$

\begin{tabular}{|c|c|c|c|c|c|c|c|c|}
\hline \multirow{2}{*}{$\begin{array}{l}\text { Constraint } \\
(\mathrm{m}, \mathrm{n})\end{array}$} & \multicolumn{2}{|c|}{ Clamped-point clamped } & \multicolumn{2}{|c|}{ Point clamped-clamped } & \multicolumn{2}{|c|}{ Free-point clamped } & \multicolumn{2}{|c|}{ Point clamped-free } \\
\hline & Present study & FE Model & Present study & FE Model & Present study & FE Model & Present study & FE Model \\
\hline$(0,0) \theta$ & 0.5148 & 0.5142 & 2.1712 & 2.1702 & 0 & 0 & 0 & 0 \\
\hline$(0,1)$ & 1.0773 & 1.0636 & 2.3021 & 2.3054 & 0.5202 & 0.5266 & 0.3251 & 0.3331 \\
\hline$(0,2)$ & 1.5554 & 1.556 & 2.6812 & 2.6685 & 1.1122 & 1.1125 & 1.1712 & 1.1732 \\
\hline$(0,3)$ & 2.1568 & 2.1616 & 3.7300 & 3.7360 & 2.0721 & 2.0738 & 2.0791 & 2.0836 \\
\hline$(1,1)$ & 2.1754 & 2.1753 & 3.3219 & 3.3249 & 1.7643 & 1.7693 & 1.6807 & 1.6849 \\
\hline
\end{tabular}

$\theta$ : pure circumferential mode.

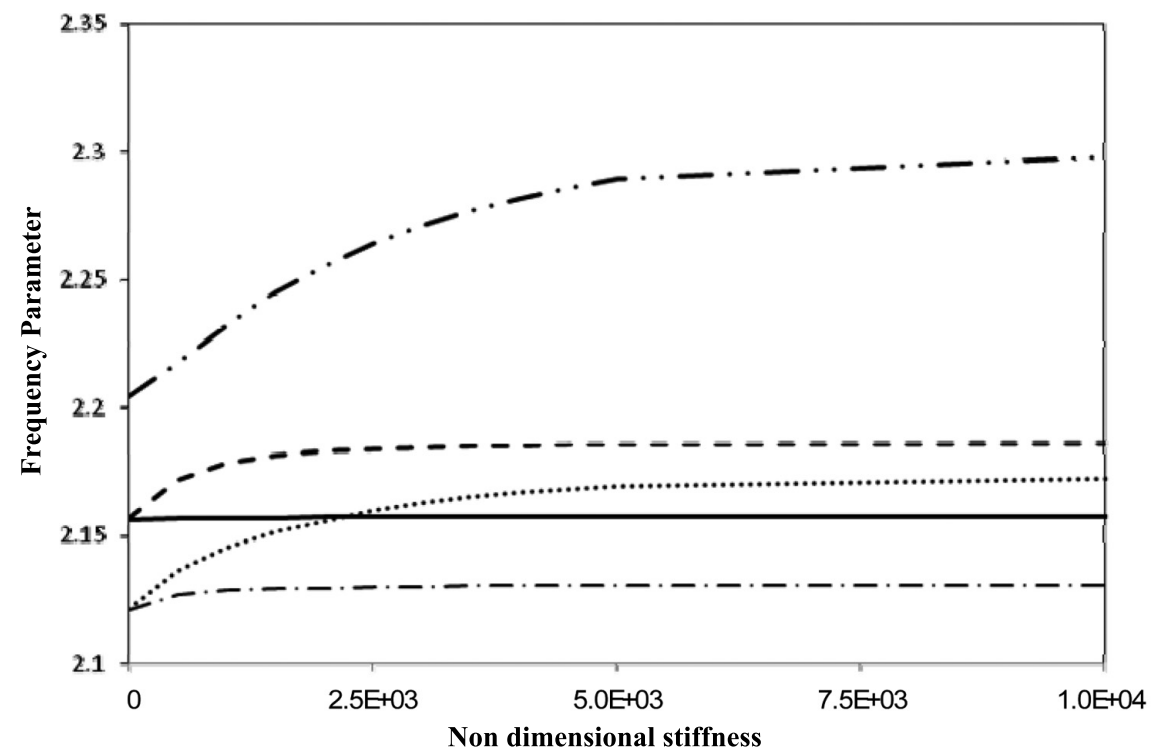

Fig. 3. Variation in the frequency parameters of selected modes with varying normalized point support stiffness at the outer edge of an annular disk clamped at the inner edge $(v=0.3, \beta=0.2),(1,1) \mathrm{E},(1,1) \mathrm{O},(0,3) \mathrm{O},(0,3) \mathrm{E},(0,0) \mathrm{E}$.

to as 'Clamped-Point Clamped' condition; (ii) Point-clamped support at the inner edge with clamped outer edge, referred to as 'Point Clamped -Clamped' condition; (iii) Free outer edge with point clamp support at inner edge, referred to as 'Point Clamped -Free' condition; and (iv) Point-clamp support at the outer edge with free inner edge, referred to as 'Free-Point Clamped' condition. A point-constraint within the annular disk is realized by introducing a two-dimensional point spring, as illustrated in Fig. 2. The analyses were performed to obtain frequency parameters of the even (coupled radial cosine and circumferential sine modes) and odd (coupled radial sine and circumferential cosine modes) subsystems corresponding to the selected modes, which are presented in Tables 3 and 4, respectively.

It is important to note that the label $(m, n)$ in Tables 3 and 4 does not reflect the nodal diameter and the nodal circle number, since the modes are coupled within each subsystem. The labels $(m, n)$ for the 'Clamped-Point Clamped' support condition, however, can be directly related to those reported in Table 1, when the point support constraint 


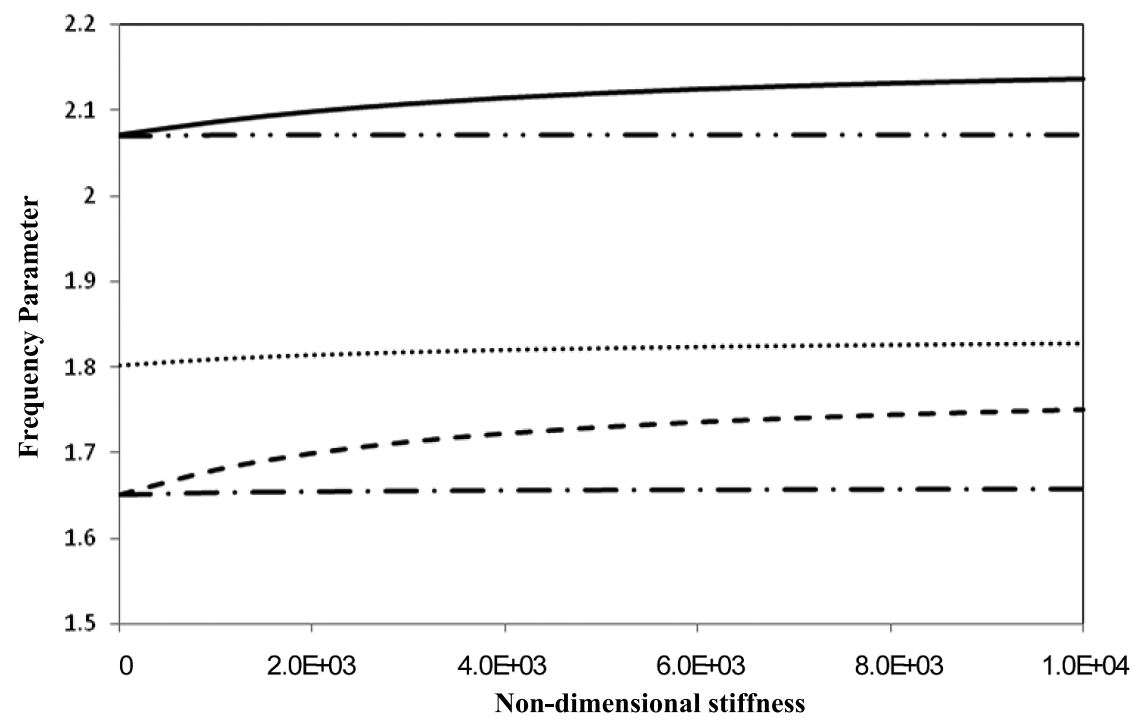

Fig. 4. Variation in the frequency parameters of selected modes with varying normalized point support stiffness at the outer edge of an annular disk free at the inner edge $(v=0.3, \beta=0.2)),(1,1) \mathrm{E},(1,1) \mathrm{O},(0,0) \mathrm{E},(0,3) \mathrm{E},(0,3) \mathrm{O}$.

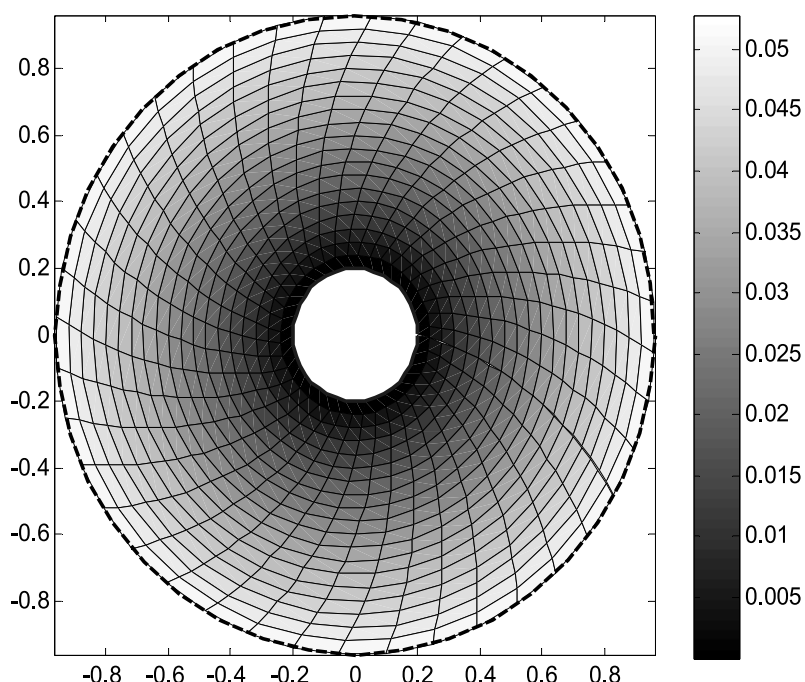

(a)

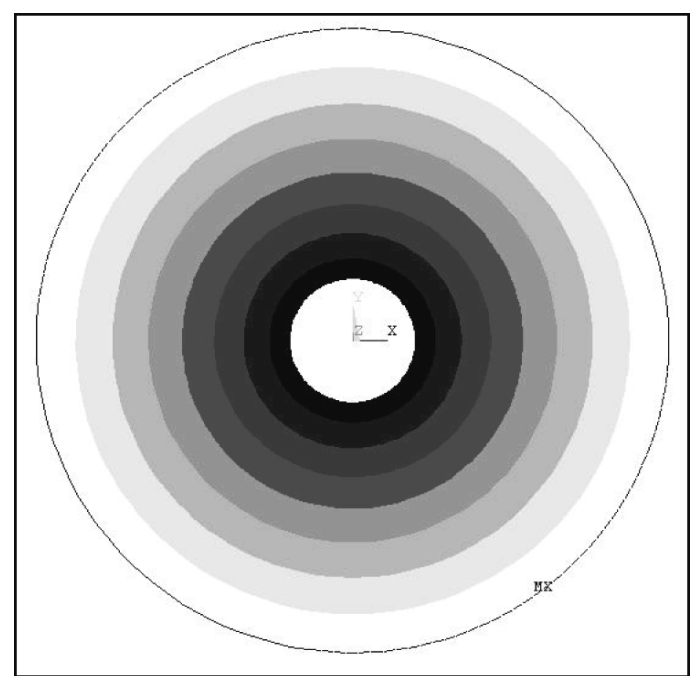

(b)

Fig. 5. Contour deformation plots for purely circumferential mode $(0,0)$ for a 'Clamped-Point Clamped' disk: a) present analysis; b) Finite element model.

is relaxed to the free edge. The results presented in Tables 1, 3 and 4 suggest that the frequency parameters of the disk with an outer edge point support are higher than those of the disk with the free outer edge. The effect of the point support is further investigated by varying the support stiffness on the frequency parameter, and the corresponding mode. The radial and circumferential stiffness parameters of the point support are varied from zero (free condition) to very large values (nearly clamped) condition. Figuer 3 illustrates the variations in the frequency parameters corresponding to selected modes with variations in the normalized stiffness parameters in the radial, $\bar{K}_{r}=K_{p r}\left(1-v^{2}\right) / E$ and circumferential, $\bar{K}_{\theta}=K_{p \theta}\left(1-v^{2}\right) / E$, directions. The figure illustrates the frequency parameters of the selected odd and even modes, denoted by ' $O$ ' and ' $E$ ', respectively. The results clearly show considerable deviation in the frequency parameters corresponding to the even and odd modes when the stiffness parameters of the point support are increased. The two, however, converge to values identical to those in Table 1 , 


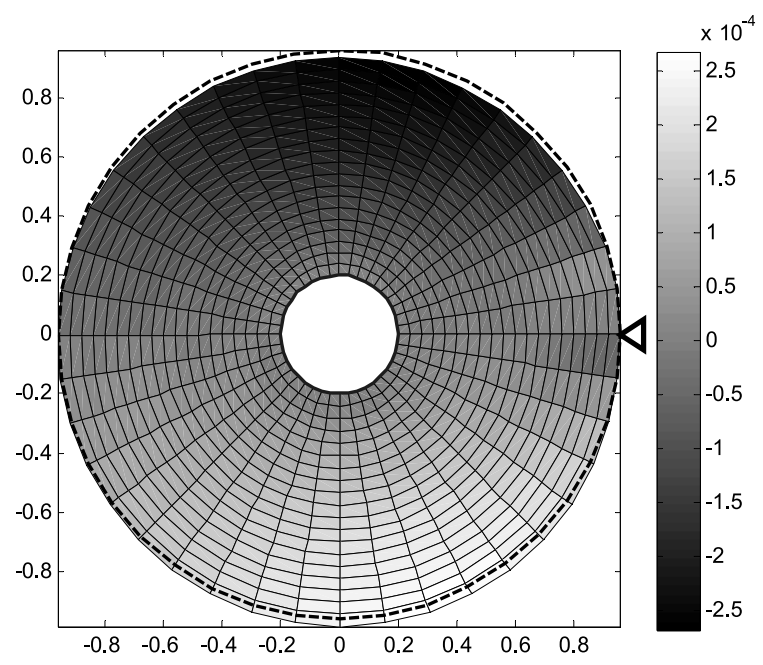

(a)

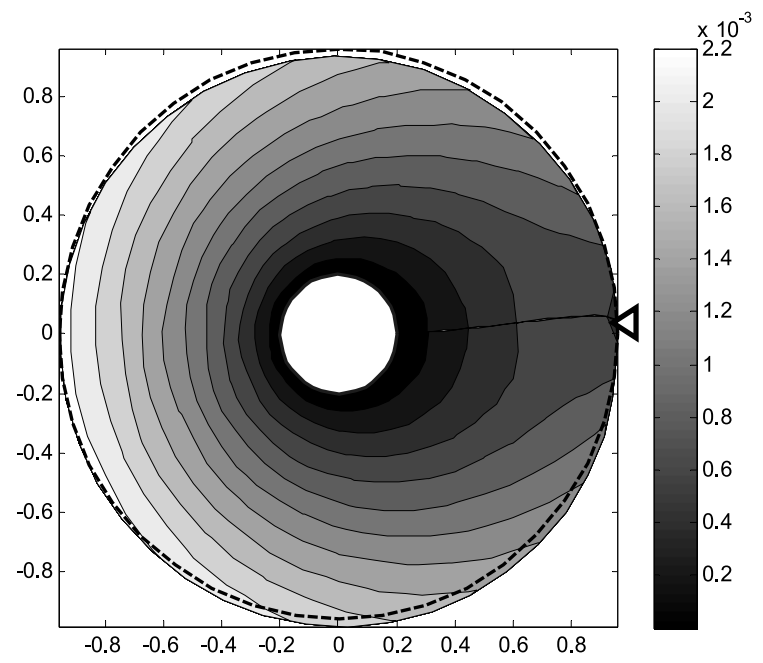

(c)

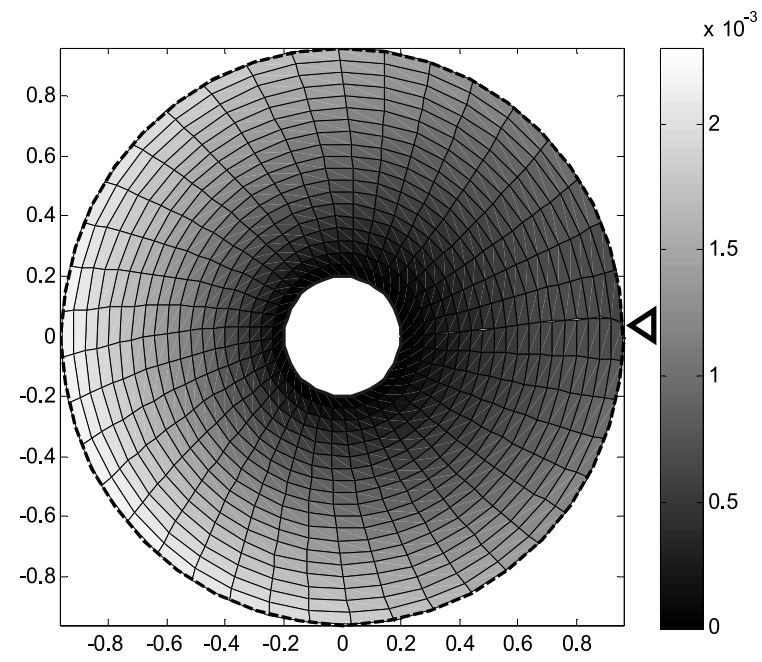

(b)

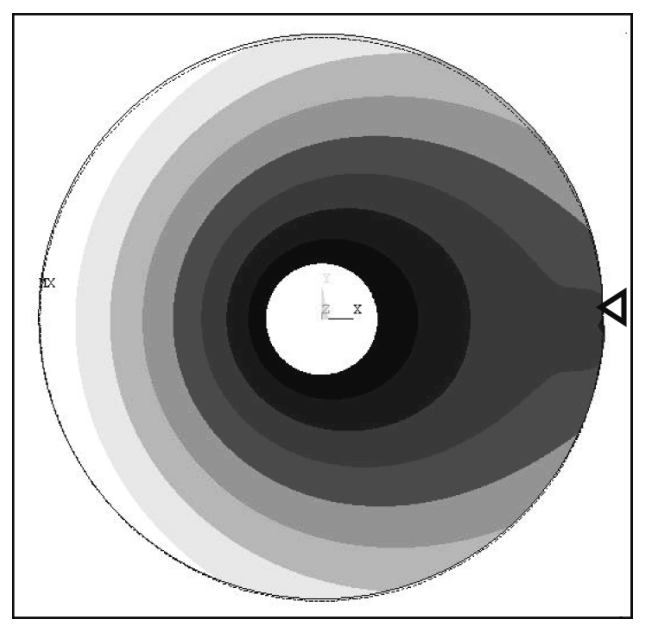

(d)

Fig. 6. Contour deformation plots for mode $(0,0)$ for a 'Clamped-Point Clamped' disk: a) radial displacement; b) circumferential displacement, c) vector sum of displacements; d) deformed shape from Finite element model, : point clamped support, undeformed edge.

when the point support stiffness diminishes to free outer edge condition. This tendency was observed for all the four constraint conditions considered. As an additional example, Fig. 4 illustrates the variations in frequency parameters of selected modes of the annular disk with free inner edge but point-supported outer edge with varying support stiffness.

The results in Fig. 3 further show a change in the order of the modes with increasing support stiffness. For the annual disk clamped at the inner edge, the frequency parameter of the $(1,1)$ odd mode becomes greater than the $(0$, 3 ) odd mode, while the order of these modes is opposite when the support stiffness is relatively small. This trend is not observed for the 'Free-Point Clamped' condition as seen in Fig. 4.

The deformation plots of in-plane vibration of the disk with different support conditions were further evaluated using Eqs (3) and (4). The results, however, are presented for the clamped inner edge and point supported outer edge in order to illustrate the effect of point clamped support on the in-plane vibration modes. Deformation contour plots for selected modes of the 'Clamped-Point Clamped' disk are presented and compared with those of the 'Clamped-Free' disk. Figure 5 illustrates the deformed shape corresponding to the lowest mode of the 'Clamped-Free' disk (mode $(0,0)^{\theta}$ in Table 1 ) obtained analytically and from the finite element analysis. This mode 


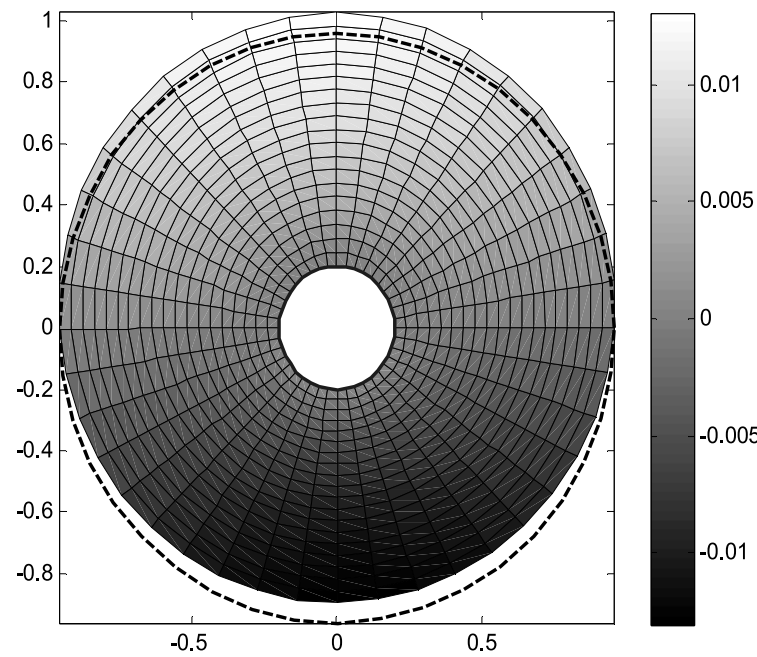

(a)

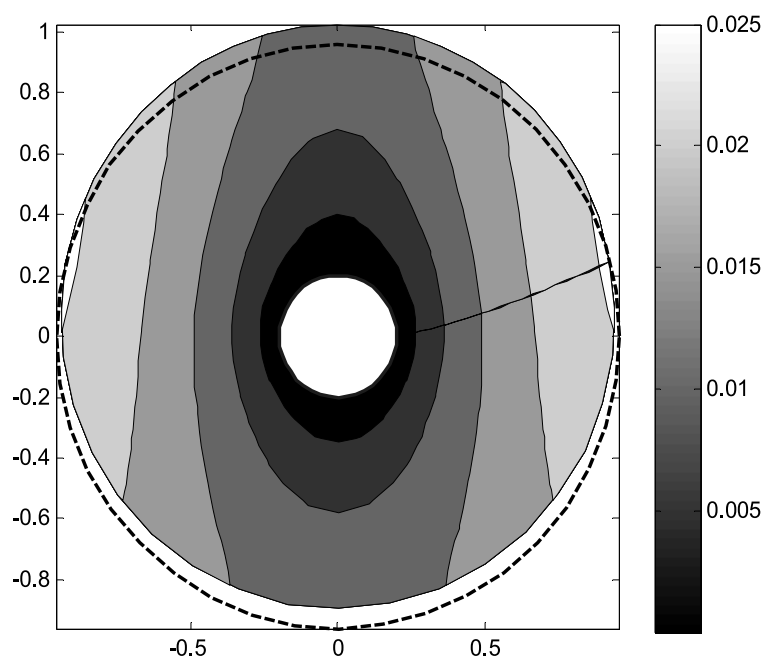

(c)

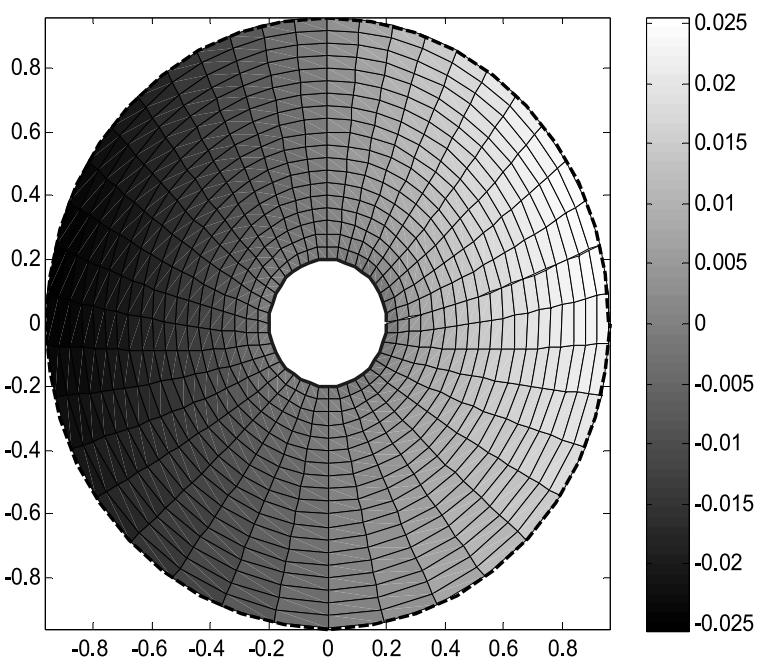

(b)

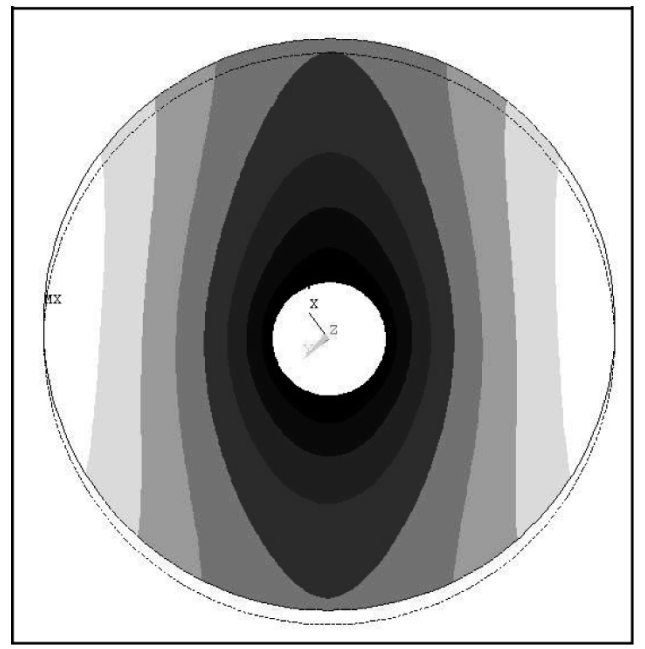

(d)

Fig. 7. Contour deformation plots for mode $(0,1)$ for a 'Clamped-Free' disk: a) radial displacement; b) circumferential displacement, c) vector sum of displacements; d) deformed shape from Finite element model.

is purely circumferential obtained from the odd subsystem. The effect of additional point clamped support at the outer edge on this mode is illustrated Fig. 6. The figure illustrates the mode shapes with predominant radial and circumferential deformation, as shown in Fig. 6(a) and 6(b), respectively, that are obtained through solution of the odd subsystem. The figure also shows the resultant deformation mode obtained from vector summation of the radial and circumferential displacements together with the deformed shape obtained from finite element analysis, as seen in Fig. 6(c) and 6(d), respectively. The results show that the addition of the point clamped support yields radial displacement apart from the circumferential deformation which makes the mode neither purely circumferential nor axisymmetric. The results also show that the resultant deformation contours corresponding to mode $(0,0)$ are quite comparable with those obtained from the finite element analysis.

From Tables 3 and 4, it can be noted that the even and odd subsystems yield distinctly different frequency parameters, with point support condition. The corresponding modes, however, exhibit predominant radial and predominant circumferential deformation. Unlike the mode $(0,0)$, the even and odd frequency parameters corresponding to non-axisymmetric modes of the 'Clamped-Free' disk are identical. The predominantly radial and circumferential deformation modes $(0,1)$ of the 'Clamped-Free' disk are illustrated in Fig. 7(a) and 7(b), respectively. These also 


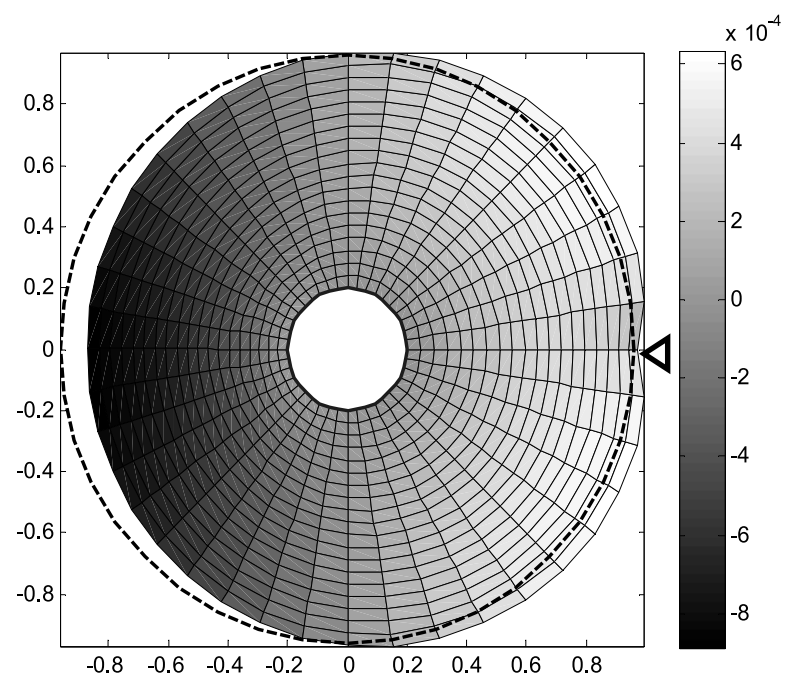

(a)

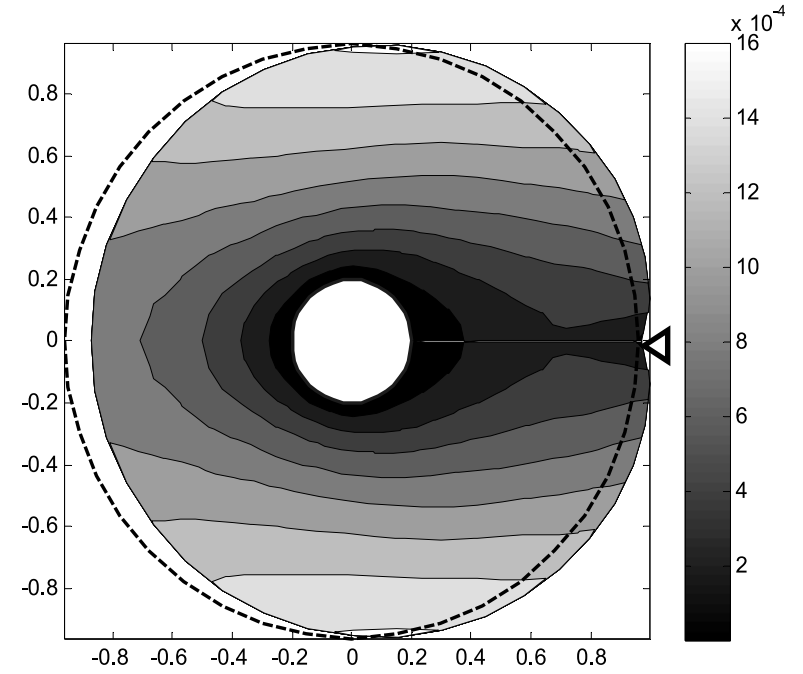

(c)

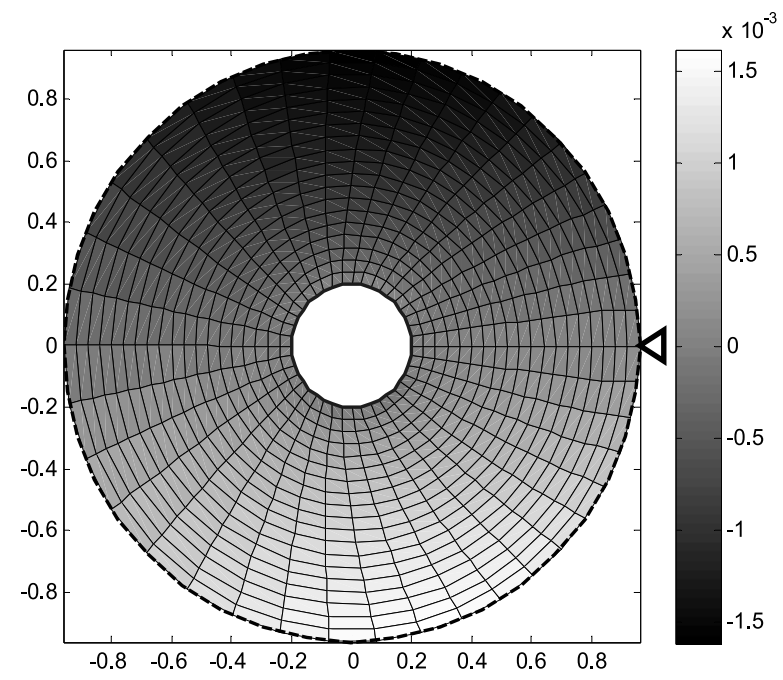

(b)

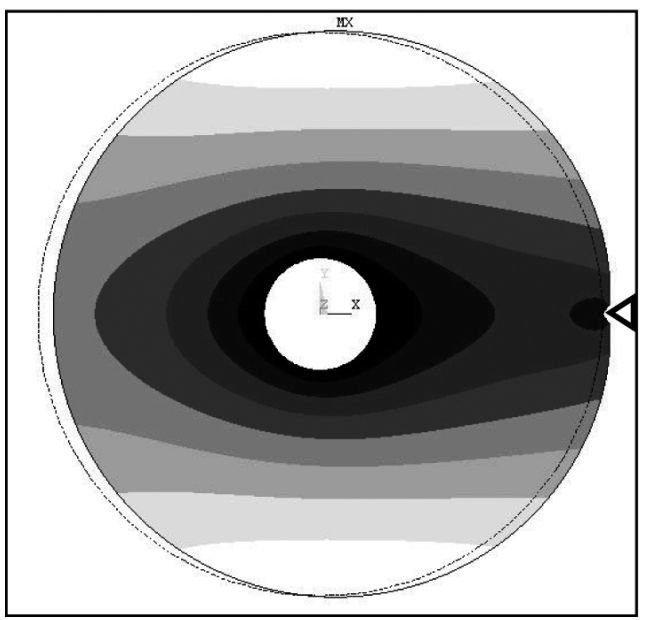

(d)

Fig. 8. Contour deformation plots for even mode $(0,1)$ for a 'Clamped-Point Clamped' disk: a) radial displacement; b) circumferential displacement, c) vector sum of displacements; d) deformed shape from Finite element model, : point clamped support , undeformed edge.

clearly show the nodal diameter in both the radial and circumferential deformation shapes. It should be noted that both the even and odd subsystems also yield identical mode shapes, except for the shift of $\pi / 2$ radians. The figure also shows the resultant deformation contours obtained from the vector summation of the radial and circumferential displacements and the finite element analysis, respectively. The addition of the outer-edge point clamped support yields a split between the odd and even frequencies, as observed in Fig. 3. The corresponding deformation modes obtained from the even and odd subsystems also differ, as seen in Figs 8 and 9 for mode $(0,1)$, respectively.

Each figure illustrates predominantly radial and circumferential deformation shapes together with the resultant deformation contours. The resultant displacement contours are quite similar to those obtained from the finite element analysis in both the cases. A comparison of Figs 7 and 8 suggests that the addition of the point clamped support does not affect the predominantly circumferential mode of the even subsystem. In a similar manner, the predominantly radial mode of the odd subsystem is insensitive to the point clamped support. This can also be deduced from Eqs (3), (4) and 2.2, which show that the even subsystem is affected only by the radial stiffness constant of the point spring at $\theta_{0}=0$, while the odd subsystem is influenced only by the circumferential stiffness of the point spring. The resultant 


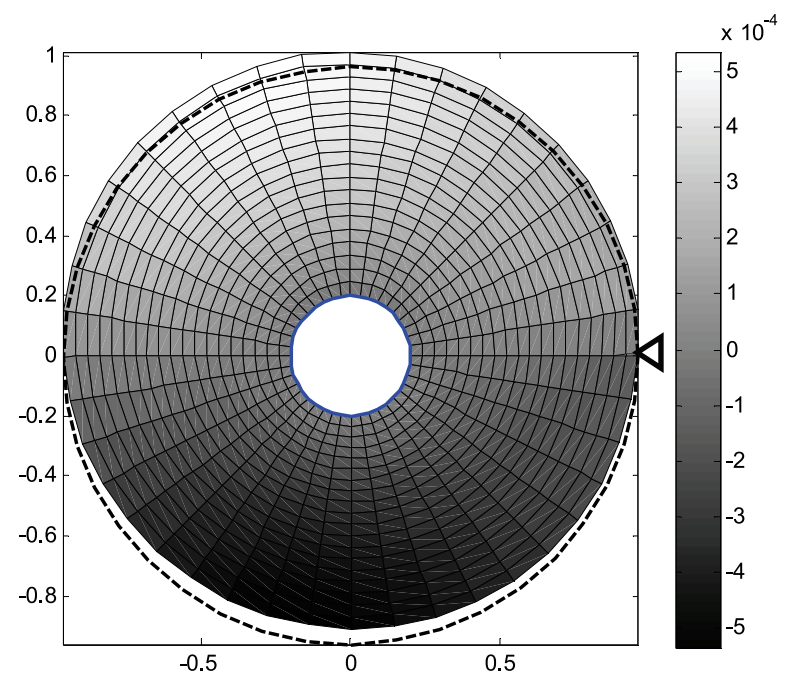

(a)

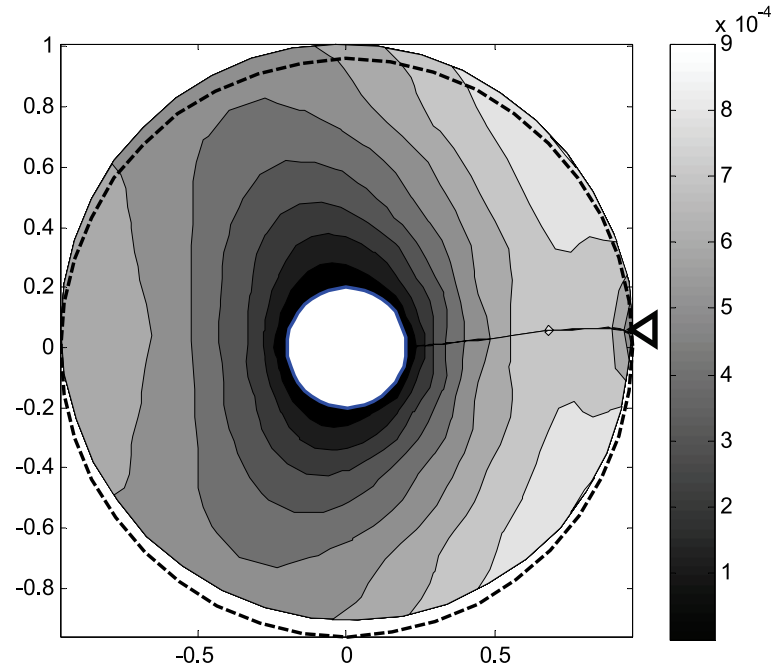

(c)

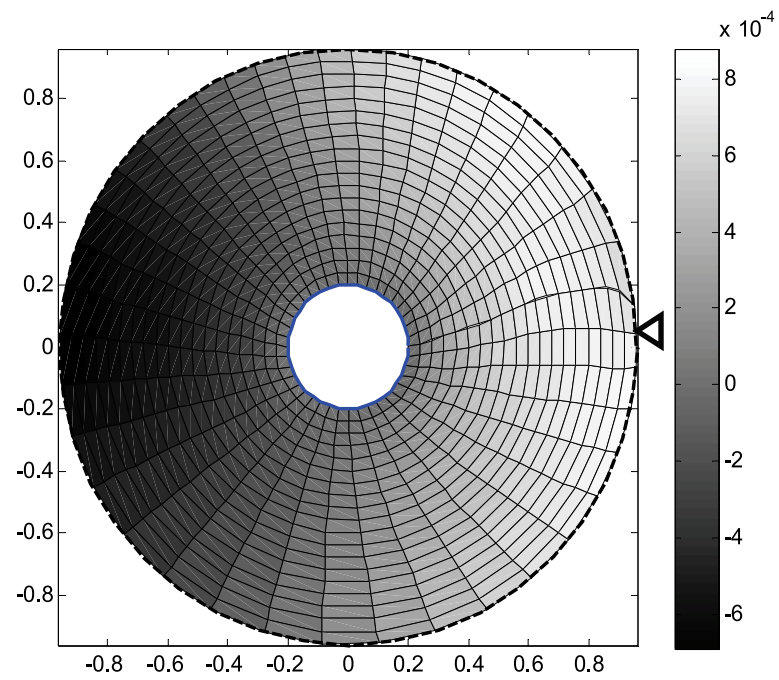

(b)

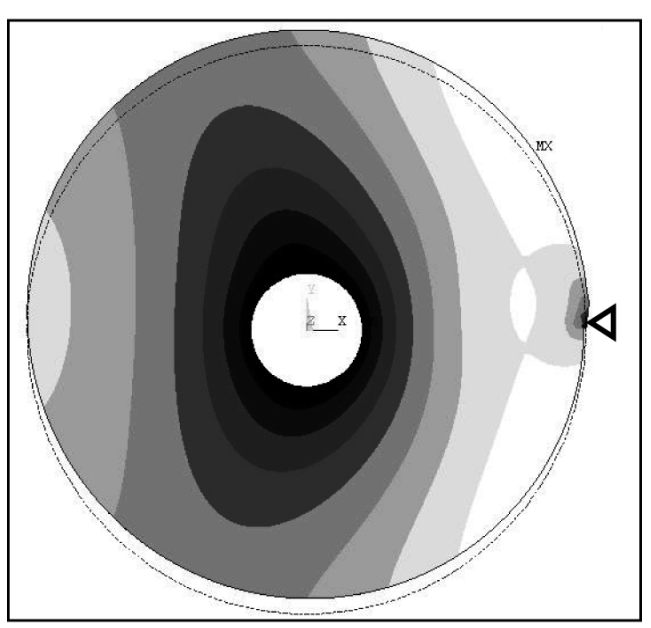

(d)

Fig. 9. Contour deformation plots for odd mode ( 0,1$)$ for a 'Clamped-Point Clamped' disk: a) radial displacement; b) circumferential displacement, c) vector sum of displacements; d) deformed shape from Finite element model, : point clamped support, undeformed edge.

even and odd subsystem in-plane vibration modes of the 'Clamped-Point Clamped' disk are affected by the radial and circumferential stiffness property of the point-support, respectively.

\section{Conclusions}

The Rayleigh-Ritz method is employed to obtain the in-plane vibration frequency parameters and associated mode shapes of an annular disk with elastic point clamped support. The displacement fields are represented by trigonometric functions in the circumferential direction and by boundary characteristics orthogonal polynomials in the radial direction. The frequency parameters are evaluated for different point-support conditions and compared with the reported data in the literature and those from finite element analysis. Due to the non-uniformity of boundary conditions, nature of coupling between the modes would differ from those observed under classical boundary conditions. It is found that partial constraints cause some frequency parameters to split into symmetric (even) 
and anti-symmetric (odd) values. The deformation plots are presented to illustrate that point clamped support has different effects on the mode shapes in the even and odd subsystems.

\section{References}

[1] K.I. Tzou, J.A. Wickert and A. Akay, In-plane vibration modes of arbitrarily thick disks, Transactions of the ASME, Journal of Vibration and Acoustics 120 (1998), 384-391.

[2] H. Lee and R. Singh, Self and mutual radiation from flexural and radial modes of a thick annular disk, Journal of Sound and Vibration 286 (2005), 1032-1040.

[3] D.J. Thompson and C.J. Jones, A review of the modelling of wheel/rail noise generation, Journal of Sound and Vibration 231 (2000), 519-536.

[4] K. Knothe and S. Grassie, Modelling of railway track vehicle/track interaction at high frequency, Vehicle System Dynamics 22 (1993), 209-262.

[5] A. Love, A Treatise on the Mathematical Theory of Elasticity, Dover Publications, New York, 1944.

[6] M. Onoe, Contour Vibrations of Isotropic Circular Plates, Journal of the Acoustical Society of America 28 (1956), 1158-1162

[7] R. Holland, Numerical studies of elastic-disk contour modes lacking axial symmetry, Journal of Acoustical Society of America 40 (1966), 1051-1057.

[8] N.H. Farag and J. Pan, Modal characteristics of in-plane vibration of circular plates clamped at the outer edge, Journal of the Acoustical Society of America 113 (2003), 1935-1946.

[9] C. Park, Frequency equation for the in-plane vibration of a clamped circular plate, Journal of Sound and Vibration 313 (2008), $325-333$.

[10] T. Irie, G. Yamada and Y. Muramoto, Natural frequencies of in-plane vibration of annular plates, Journal of Sound and Vibration 97 (1984), $171-175$.

[11] G. Ambati, J.F.W. Bell and J.C.K. Sharp, In-plane vibrations of annular rings, Journal of Sound and Vibration 47 (1976), $415-432$.

[12] K. Singa Rao and C.L. Amba-Rao, Lateral vibration and stability relationship of elastically restrained circular plates, AIAA 10 (1972), 1689-1690.

[13] P.A.A. Laura, J.C. Paloto and R.D. Santos, A note on the vibration and stability of a circular plate elastically restrained against rotation, Journal of Sound and Vibration 41 (1975), 177-180.

[14] P.A.A. Laura, L.E. Luisoni and J.J. Lopez, A note on free and forced vibrations of circular plates: the effect of support flexibility, Journal of Sound and Vibration 47 (1976), 287-291.

[15] C.S. Kim and S.M. Dickinson, The flexural vibration of thin isotropic and polar orthotropic annular and circular plates with elastically restrained peripheries, Journal of Sound and Vibration 143 (1990), 171-179.

[16] Y. Narita and A.W. Leissa, Flexural vibrations of free circular plates elastically constrained along parts of the edge, International Journal of Solids and Structures 17 (1981), 83-92.

[17] A.W. Leissa, P.A.A. Laura and R.H. Gutierrez, Transverse vibrations of circular plates having nonuniform edge constraints, Journal of Acoustical Society of America 66 (1979), 180-1184.

[18] P.A.A. Laura and G.M. Ficcadenti, Transverse vibrations of circular plates of varying thickness with non-uniform edge constraints, Applied Acoustics 13 (1980), 227-236.

[19] M. Amabili, R. Pierandrei and G. Frosali, Analysis of vibrating circular plates having non-uniform constraints using the modal properties of free-edge plates: application to bolted plates, Journal of Sound and Vibration 206 (1997), 23-38.

[20] R.B. Bhat, Natural frequencies of rectangular plates using characteristic orthogonal polynomials in Rayleigh-Ritz Method, Journal of Sound and Vibration 102 (1985), 493-499.

[21] V. Srinivasan and V. Ramamurti, Dynamic response of an annular disk to a moving concentrated, in-plane edge load, Journal of Sound and Vibration 72 (1980), 251-262.

[22] S.M. Bashmal, R. Bhat and S. Rakheja, In-plane free vibration of circular disks using characteristic orthogonal polynomials in Rayleigh-Ritz method, Canadian Acoustics 35 (2007), 164-165.

[23] F.E. Eastep and F.G. Hemmig, Natural frequencies of circular plates with partially free, partially clamped edges, Journal of Sound and Vibration 84 (1982), 359-370. 

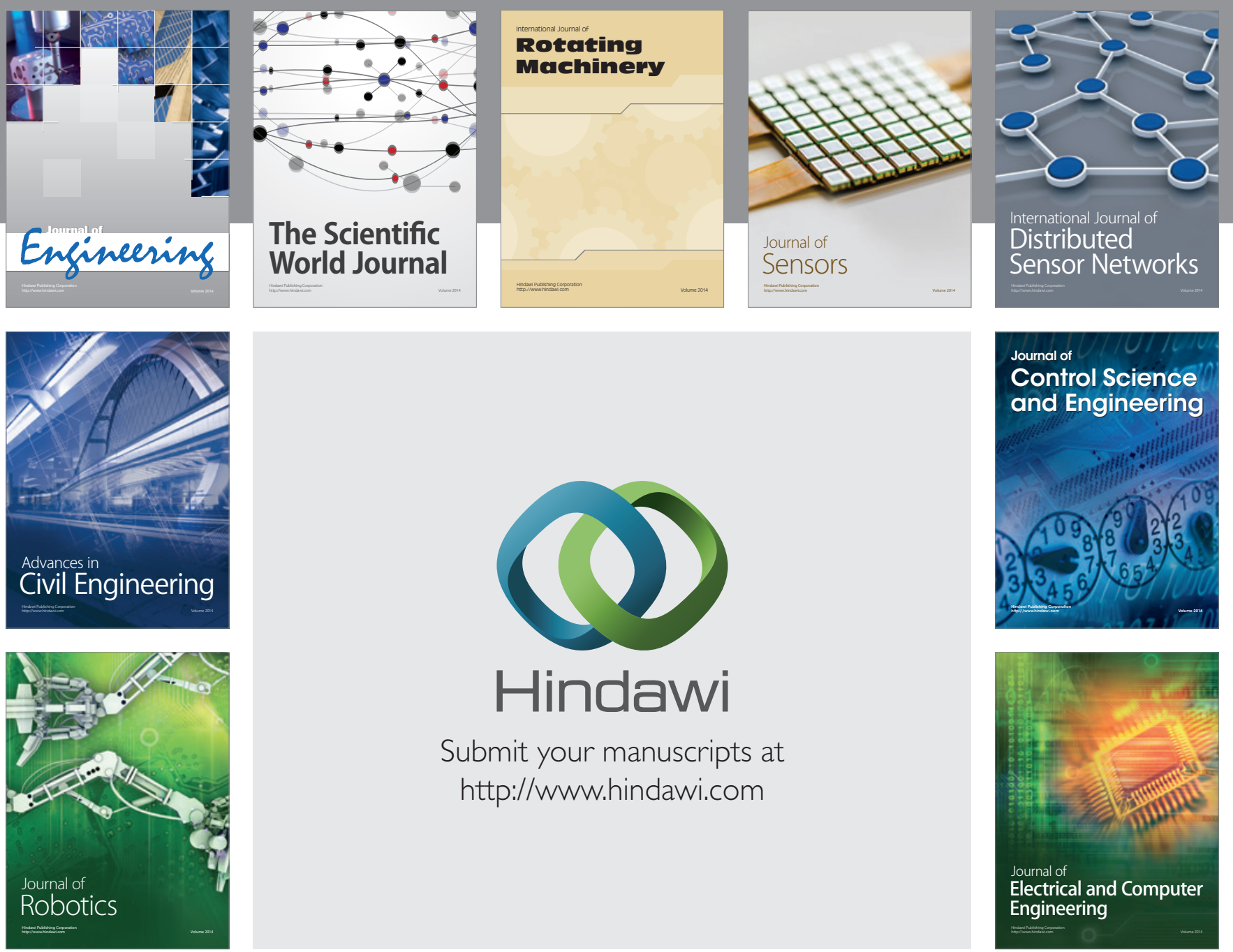

Submit your manuscripts at

http://www.hindawi.com
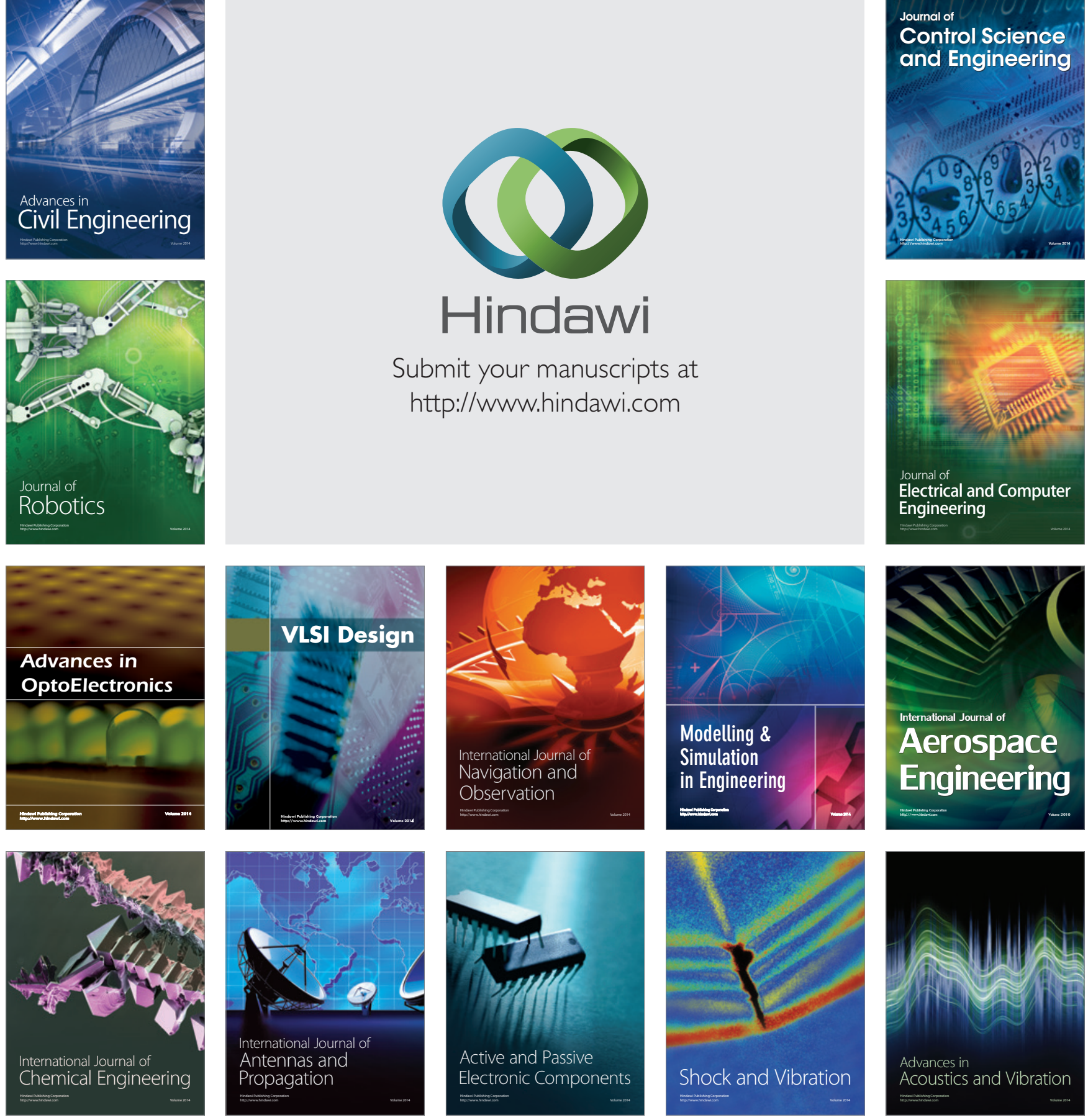